\title{
Criminologie
}

\section{Police, État, insécurité}

\section{René Lévy et Philippe Robert}

Volume 17, numéro 1, 1984

La police après 1984

URI : https://id.erudit.org/iderudit/017190ar

DOI : https://doi.org/10.7202/017190ar

Aller au sommaire du numéro

Éditeur(s)

Les Presses de l'Université de Montréal

ISSN

0316-0041 (imprimé)

1492-1367 (numérique)

Découvrir la revue

Citer cet article

Lévy, R. \& Robert, P. (1984). Police, État, insécurité. Criminologie, 17(1), 43-58. https://doi.org/10.7202/017190ar d'utilisation que vous pouvez consulter en ligne.

https://apropos.erudit.org/fr/usagers/politique-dutilisation/ 
La rareté des travaux sociologiques sur la police en France laisse le champ libre à une littérature de témoignage, de polémique ou de reportage - d'ailleurs éventuellement intéressante - qui facilite la production quasiment exclusive de discours très globaux, qui obscurcissent le problème plutôt que d'en éclairer l'analyse. Cette situation est encore aggravée par le nombre limité des travaux historiques sérieux sur la police, au moins pour l'époque «contemporaine» $\mathrm{XIX}^{c}-\mathrm{XX}^{e}$ siècles) ${ }^{1}$.

Dans cette situation, on ne peut certes échapper au raisonnement conjectural mais on peut au moins prendre la précaution d'en asseoir les prémisses sur les thèmes que l'analyse sociologique a déjà entrepris d'explorer.

Par ailleurs, notre propos n'est pas ici de jeter les bases d'une sociologie globale de la police en France; nous allons limiter nos réflexions à l'aspect pénal de ses fonctions, qui est celui dont l'abord est le plus aisé, en raison du relatif développement, dans ce pays, d'une sociologie des matières pénales.

Une sociologie de la police ne peut être enfermée dans la spécificité de son objet, sinon elle sombre dans le fonctionnalisme, ou verse dans la paranoïa qui croit y lire tout ce qu'il y a d'important et de caractéristique dans l'évolution contemporaine de nos formations sociales. Il faut tenter, sans diluer l'objet d'en briser la clôture. Ainsi essayons-nous ici d'articuler la caractéristique, actuellement déterminante, du rôle pénal de la police (la disjonction croissante entre le contentieux enregistré et celui qui est traité), avec le débat sur le sentiment d'insécurité et la manière dont l'État tente de le gérer.

$A$ la fois pour des raisons internes - commodités corporatistes - et externes - urgence et difficulté de la régulation de fonctions collectives généralisées, comme la circulation routière ou l'usage du chèque - les efforts de la police ont été concentrés sur d'autres contentieux que les plaintes des particuliers, au moment même où cellesci s'accroissaient fortement; et cette contradiction a autorisé, nous

* Service d'études pénales et criminologiques, Paris (LA.-C.N.R.S. 313).

1. Pour une synthèse des travaux historiques sur le pénal en France, voir Robert et Levy (s.p.). La bibliographie la plus complète sur l'histoire de la police française est Le Clere (1980). 
semble-t-il, la cristallisation sur le crime, du mécontentement ou de l'insécurité de plusieurs fractions sociales.

En se fondant sur l'analyse de statistiques et sur les résultats de certaines recherches, on dégagera un certain nombre de caractéristiques de la pratique policière et de ses résultats qui sont suffisamment attestées. Puis, à titre d'hypothèses en vue de travaux à venir, on tentera de dessiner une liaison entre ces constatations et l'actuel débat sur l'«insécurité».

\section{LES ORIENTATIONS DE LA PRATIQUE POLICIËRE}

S'il existe peu de recherches d'ensemble sur la police, on dispose au moins d'une statistique administrative, publiée par le ministère de l'Intérieur depuis $1972^{2}$. Cette statistique ne porte que sur les affaires pour lesquelles la police adresse finalement un procès-verbal au ministère public (c'est une statistique des extrants et non des intrants). Elle totalise l'activité des deux sortes de police existant en France: la police nationale, plutôt urbaine, et la gendarmerie nationale, plutôt rurale. Enfin, elle exclut — paradoxalement - la majorité de l'activité répressive de la police: les procès-verbaux pour les petites infractions (contraventions) et même les procès-verbaux de certains délits: ceux qui concernent des infractions aux règles de circulation ou des atteintes par imprudence à la personne humaine ${ }^{3}$. De cette statistique nous allons retenir trois renseignements: le nombre des affaires enregistrées, celui des affaires "élucidées»", et celui des personnes mises en cause. Nous présentons ces données dans les tableaux 1 et 2 qui montrent l'un, leur ventilation selon les sortes d'infraction, et l'autre, leur évolution sur plusieurs années.

L'observation de ces séries fait apparaître une double discordance, d'une part entre les affaires enregistrées et les affaires élucidées, d'autre part entre le nombre des affaires enregistrées et le nombre des mises en cause. Et ces discordances vont croissant.

2. De 1949 à 1972, il existe seulement une statistique non publiée et d'ailleurs beaucoup plus sommaire. Pour la période antérieure on trouve seulement quelques éléments sur les effectifs et les affaires traitées dans le Compte général de la Justice criminelle, publiée depuis 1825 par le ministère de la Justice.

3. En 1981, la statistique policière a enregistré quelque 3 millions de procèsverbaux, alors que les seules infractions de circulation, qu'elle n'enregistre pas, approchent 14 millions (Direction de la Réglementation, inédit).

4. La police considère comme élucidée une af faire pour laquelle elle a réussi à mettre en cause au moins un suspect. 
TABLEAU 1

Statistiques de police et de gendarmerie (Faits enregistrés et élucides), 1981.

\begin{tabular}{|c|c|c|c|c|c|}
\hline Catégories d'infractions & $\begin{array}{l}\text { Nombre } \\
\text { de fatss } \\
\text { enregiatres }\end{array}$ & $\%$ & $\begin{array}{l}\text { Nombre } \\
\text { de faits } \\
\text { élucides }\end{array}$ & $\%$ & $\begin{array}{l}\text { Taux } \\
\text { d'éluci- } \\
\text { dation }\end{array}$ \\
\hline Atteintes à la personne & $\begin{array}{l}50079 \\
19146\end{array}$ & $\begin{array}{l}1,73 \\
0,66\end{array}$ & $\begin{array}{l}37806 \\
14635\end{array}$ & 3,19 & $\begin{array}{l}75,49 \\
76,44\end{array}$ \\
\hline Infractions en matière & & & & & \\
\hline familiale et sanitaire & 49972 & 1,73 & 44746 & 3,76 & 89,54 \\
\hline $\begin{array}{l}\text { Infractions en matière d'ordre } \\
\text { public et de réglementation }\end{array}$ & 86251 & 2,98 & 75426 & 6,37 & 87,45 \\
\hline $\begin{array}{l}\text { Infractions astucieuses contre } \\
\text { les biens }\end{array}$ & 274120 & 9,49 & 216445 & 18,28 & 78,45 \\
\hline $\begin{array}{l}\text { Infractions en matière de } \\
\text { chèques }\end{array}$ & 337426 & 11,68 & 313 & 26,51 & 93 \\
\hline Vols avec violence & 43242 & 1,50 & 11400 & 0,96 & 26,36 \\
\hline Cambriolages & 308122 & 10,66 & 47376 & 4 & 15,38 \\
\hline Vols de et dans les véhicules & & & & & \\
\hline à mote & 928649 & 32,13 & 100997 & 8,53 & 10,88 \\
\hline $\begin{array}{l}\text { Vols à l'étalage } \\
\text { Autres vols et recels }\end{array}$ & $\begin{array}{r}74265 \\
404580\end{array}$ & 2,57 & $\begin{array}{l}58964 \\
77196\end{array}$ & $\begin{array}{l}4,98 \\
6,52\end{array}$ & $\begin{array}{l}73,40 \\
19,08\end{array}$ \\
\hline fractions contre & & & & & \\
\hline les biens & 165680 & 5,73 & 28025 & 2,36 & 16,92 \\
\hline Divers & 148488 & 5,14 & 157067 & 13,27 & \\
\hline TOTAL & 2890020 & 100 & 1183622 & 100 & 40,96 \\
\hline
\end{tabular}

Source: Intérieur, 1983, 63 à 65.

La statistique des faits enregistrés offre une image presque entièrement occupée par les atteintes aux biens. Les infractions contre les personnes, les mœurs, la famille, l'ordre public et la réglementation ne totalisent qu'environ $7 \%$, et parmi le reste, les infractions dites astucieuses ne représentent qu'environ $9 \%$. Il n'y a donc guère dans cette statistique que des vols de toute sorte et, bien loin derrière, des affaires de chèques. Si l'on regarde plus en détail, on est frappé par l'importance du contentieux lié aux véhicules à moteur: la moitié des vols concerne soit des véhicules à moteur, soit des objets pris dans des voitures. Les infractions en matière de chèques retiennent ensuite l'attention avec quelque $12 \%$ au total ${ }^{5}$. Les vols simples atteignent un ordre de grandeur analogue et les cambriolages suivent de près avec $10 \%$. En échange, les vols avec violence ne représentent que $1,5 \%$ du total des faits enregistrés.

5. Il s'agit surtout de l'émission de chèques sans provision préalable et suffisante qui constitue en France, une infraction. 
TABLEAU 2

Série corrigée des statistiques de police judiciaire (Police + Gendarmerie), 1950-1981

\begin{tabular}{|c|c|c|}
\hline Années & Affaires & Mis en cause \\
\hline $\begin{array}{r}1950 \\
51 \\
52 \\
53 \\
54 \\
55 \\
56 \\
57 \\
58 \\
59 \\
60 \\
61 \\
62\end{array}$ & $\begin{array}{l}404233 \\
371 \quad 693 \\
493295 \\
392762 \\
387881 \\
388279 \\
401 \quad 469 \\
482873 \\
494305 \\
532558 \\
568984 \\
597918 \\
625291\end{array}$ & $\begin{array}{l}235837 \\
218847 \\
221 \quad 736 \\
216875 \\
209545 \\
204 \quad 241 \\
202750 \\
229707 \\
228116 \\
229451 \\
226241 \\
115996 \\
237796\end{array}$ \\
\hline $\begin{array}{l}63 \\
64 \\
65 \\
66 \\
67 \\
68 \\
69 \\
70 \\
71\end{array}$ & $\begin{array}{r}645695 \\
692641 \\
747469 \\
830546 \\
946176 \\
1055557 \\
1148009 \\
1274368 \\
1445639\end{array}$ & $\begin{array}{l}314765 \\
341744 \\
366612 \\
399016 \\
443641 \\
465296 \\
535868 \\
608966 \\
650981\end{array}$ \\
\hline $\begin{array}{l}72 \\
73 \\
74 \\
75 \\
76 \\
77 \\
78 \\
79 \\
80 \\
81\end{array}$ & $\begin{array}{lll}1 & 675 & 507 \\
1 & 763 & 372 \\
1 & 827 & 373 \\
1 & 912 & 327 \\
1 & 823 & 953 \\
2 & 097 & 919 \\
2 & 147 & 832 \\
2 & 330 & 566 \\
2 & 627 & 508 \\
2 & 890 & 020\end{array}$ & $\begin{array}{ll}727 & 154 \\
753 & 488 \\
717 & 116 \\
682 & 132 \\
515 & 517 \\
582 & 770 \\
574 & 937 \\
609 & 669 \\
686 & 354 \\
746 & 893\end{array}$ \\
\hline
\end{tabular}

Source: Intérieur, 1950-1971 (tableaux ronéotés non diffusés), 1972-1983.

En fin de compte, les faits enregistrés sont surtout:

- des vols de et dans les véhicules à moteur;

- des infractions relatives aux chèques;

- des vols simples;

- des cambriolages, dont l'importance contraste avec la faiblesse du contentieux de violence physique.

La distribution des faits élucidés fait une part relative plus importante aux atteintes à la personne et aux mœurs, aux infractions 
en matière familiale et sanitaire, à celles concernant l'ordre public et la réglementation, aux infractions "astucieuses», à celles de chèques, au vol à l'étalage. En revanche, elle réserve une part moindre au cambriolage, aux «autres vols», aux «autres infractions contre les biens» et surtout aux vols de et dans les véhicules.

En définitive, ce sont donc surtout les différentes sortes de vols au détriment de particuliers qui sont minorés dans la distribution des mises en cause, si on la compare à celle des faits enregistrés.

Les taux d'élucidation fournissent la clé du mystère: le taux moyen d'élucidation est de 2 faits sur 5 , mais il cache deux cas de figure différents. Il tourne autour de 9 sur 10 pour les affaires de chèques, les infractions familiales et sanitaires et celles concernant l'ordre public; et encore autour des $3 / 4$ pour les infractions «astucieuses», les atteintes aux mours ou à la personne et les vols à l'étalage.

D'un autre côté, il oscille entre 1 sur 10 (vol de et dans les véhicules) et 1 sur 4 (vol avec violence), avec des cas de figure intermédiaires (cambriolage, «autres atteintes aux biens», «autres vols»). De là, la forte différence observée entre les deux premières distributions.

En fait, la statistique policière présente comme un tout deux ensembles qui ne sont que très partiellement sécants.

Le premier ensemble est constitué par les faits que des plaignants portent à la connaissance de la police. Les atteintes aux biens y prédominent ${ }^{6}$, ne serait-ce que parce que le comportement de plainte est dans ce cas déterminé par des considérations extérieures au processus pénal'; deux d'entre elles sont particulièrement importantes:

- le développement de l'assurance de toutes sortes de biens qui entraîne l'obligation - imposée par les compagnies - de déposer une plainte avant de déclarer le sinistre ${ }^{8}$,

6. Voyez par exemple: Barberger-Damamme (1981).

7. Que celles-ci jouent un rôle dans le déclenchement de la plainte ne signifie pas que les attentes qui accompagnent ce comportement se réduisent à elles.

8. Ce prérequis a pour objet de limiter les déclarations frauduleuses; mais dans la mesure où les plaignants savent que leurs plaintes ne seront pas suivies d'enquête la plupart du temps, la garantie fournie aux assurances par l'intervention policière est illusoire; cette situation génère même vraisemblablement des plaintes imaginaires ou excessives dans un but de fraude, les plaignants sachant que la police authentifiera sans difficulté leurs déclarations. Pour des exemples, voir Bonnemain $(1978$, p. 91) et Berberger-Damamme (1981, p. 497). 
- le développement de biens immatriculés - c'est-à-dire nominativement rattachés à leurs propriétaires - et dont l'usage par le voleur pourrait engager la responsabilité du propriétaire s'il ne manifestait pas officiellement que le bien en question lui a été soustrait ${ }^{9}$.

Ce premier ensemble est massivement enregistré dans la statistique des faits enregistrés, dans la mesure où, comme on le sait, l'approvisionnement de la police est beaucoup plus réactif que proactif ${ }^{10}$.

Le second ensemble est constitué par les produits de l'initiative policière. Appréciée par rapport à l'ensemble des affaires enregistrées, dans lequel les procédures contre inconnu, qui n'aboutissent quasiment jamais, pèsent d'un poids énorme, cette initiative compte peu. Mais si on l'examine du point de vue des affaires qui aboutissent, le résultat est tout différent ${ }^{11}$. Massivement, l'orientation de l'initiative policière est déterminée par des raisons toutes différentes de celles qui commandent le comportement de plainte. Joue d'abord un rôle fondamental cet objectif premier des missions policières qu'est le maintien de l'ordre dans les lieux publics, lequel comprend particulièrement, à l'époque actuelle, la police de la circulation routière. En outre, l'intervention policière est plus aisée dans la rue ou dans les lieux publics que dans des lieux privés.

S'ajoutent à cela les priorités officielles de la répression à tel ou tel moment, 1'incidence de l'allocation des ressources ${ }^{12}$ et celle de l'évaluation de la productivité des services ${ }^{13}$.

9. Ả l'instar des compagnies d'assurance, les administrations (pour les documents d'identité et les véhicules à moteur) et les banques (pour les chéquiers et les cartes de crédit) exigent un dépôt de plainte préalable. Voir Serverin et Bruxelles (1981, p. 140 et s.)

10. Barberger-Damamme (1981, p. 523).

11. En ce sens Levy (1982, p. 41).

12. Dans une police où existe une stricte division entre personnels d'exécution (personnels en «tenue») et personnels d'enquête (police judiciaire, en «civil»), la priorité donnée à ceux-là influe sur la capacité d'enquête. En outre, l'affectation des personnels entre ces différents services n'est pas sans incidence: témoin, d'une part le développement d'unités spécialisées qui favorisent l'activité proactive aux dépens de l'enquête sur plainte, ou bien les très faibles effectifs attribués aux services chargés de traiter les plaintes (commissariats de police locaux). Pour un exemple particulier sur ce point, voir Barberger-Damamme (1981, p. 414), ou encore les effets du regroupement des effectifs en «Hôtels de police» urbains centralisés plutôt qu'en implantations dispersées.

13. La recherche d'une rentabilité mesurée en nombre de mise en cause («faire un crâne» dans le jargon policier français) favorise la démarche proactive aux dépens du travail souvent ingrat et peu productif d'enquête sur plainte. 
La statistique des mises en cause rend massivement compte de ce second ensemble.

Quant à la statistique des affaires élucidées, elle montre la faiblesse relative des plages de recouvrement entre les deux ensembles.

En effet, pour que se rencontrent le comportement de plainte et celui d'enquête, il faut se situer dans l'un des deux cas suivants.

- Ou bien la plainte déclenche une enquête, ce qui - hors les cas d'affaires jugées très graves - n'a de chance de se produire en pratique que si le plaignant fournit des éléments d'identification d'un suspect ${ }^{14}$. Or, la très grande majorité des plaintes relatives à des vols ou des cambriolages excluent tout élément de ce genre. On observe d'ailleurs que, tout en restant toujours très faible, le taux d'élucidation augmente quand on passe de vols totalement anonymes (vols de véhicules à moteur) à des vols où la victime rencontre forcément l'auteur (vol avec violence). De surcroît, les contentieux où la plainte s'accompagne plus fréquemment de l'identification d'un suspect - par exemple les coups et blessures volontaires - sont aussi ceux où l'on observe le plus fréquemment des signalements à la police sans dépôt formel de plainte. C'est justement parce que la démarche de la victime auprès de la police s'intègre souvent dans un processus complexe d'interactions et de négociations entre les protagonistes. La définition des statistiques policières françaises exclut tous ces cas, où n'intervient finalement pas une procédure officielle ${ }^{15}$.

- Ou bien, des enquêtes entreprises d'initiative par la police et enregistrées comme ayant abouti à des mises en cause, correspondent à des affaires ayant par ailleurs fait l'objet de plainte. Comme le montre Levy ${ }^{16}$, ce cas de figure se rencontre surtout dans les cas d'infractions sur la voie et dans les lieux publics (par ex. le vol à la tire ou le vol dans les véhicules). L'observation des taux d'élucidation montre néanmoins que cette seconde hypothèse est également rare, du moins en valeur relative.

14. C'est le cas pour le seul contentieux de vol où le taux d'élucidation est élevé $(75 \%)$ : le vol à l'étalage, où la victime, quand elle appelle la police a généralement identifié et même saisi le suspect. Voir Faugeron, Fichelet, Robert $(1977$, p. 131 et s.) et Zauberman (1982a, p. 49 et s., et 1981b, notamment p. 36).

15. Ils sont seulement mentionnés dans des registres dits de «main-courante» qui ont notamment été dépouillés par Bonnemain (1978), dont les données ont été réanalysées par Levy (1983), chap. I, section 2).

16. Levy (1982, p. 41). 
On se trouve donc en face d'un divorce tendanciel entre ce que l'on signale à la police et ce qu'elle traite effectivement.

Plus exactement, tout se passe comme si l'on s'orientait - bien entendu sans que les agents en aient le propos ou même une conscience claire - vers une division entre trois modes de gestion des illégalismes, selon, en particulier, le lieu de commission. On distinguerait ainsi:

- les infractions commises sur la voie ou dans des lieux publics, gérées par la police officielle;

- les infractions commises dans des lieux privés accessibles au public, gérées par des services de sécurité privée ${ }^{17}$;

- les infractions commises dans des lieux privés non accessibles au public, dont la gestion serait renvoyée à une combinaison de l'industrie de protection et de l'assurance (outre une intervention marginale des services de sécurité privés).

Cette tendance à une répartition des rôles semble soulever de sérieuses résistances et c'est dans ses conséquences sur la protection

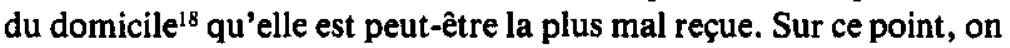
ne possède malheureusement pas encore de données extensives, si ce n'est la persistance de la controverse sur l'insécurité; à cet indice $\alpha$ contrario, on peut ajouter des indications parcellaires fournies par certaines enquêtes.

Le couple industrie de protection-assurance peut faire l'objet, en effet, de trois sortes de critiques ${ }^{19}$. On lui reproche d'abord son inefficacité relative: soit qu'il règne un certain scepticisme sur la capacité des mesures de protection à prévenir les infractions, soit que les remboursements par les assurances soient jugés insuffisants ou trop compliqués à obtenir.

Une deuxième critique porte sur le coût rapidement croissant de ses prestations (et sur l'effet multiplicateur que produisent les exi-

17. Sans que ces services aient en France atteint le développement qu'ils connaissent en Amérique du Nord (voir, par exemple Shearing et Stenning, 1983), leur croissance est un des phénomènes marquants de la dernière décennie (voir Godef roy et Laffargue, 1982).

18. Entendu largement, comme pouvant s'étendre pour certains à leur véhicule garé à proximité, et pour d'autres à leur local professionnel, commercial ou artisanal.

19. Relevées, par exemple, dans une campagne d'entretiens rétrospectifs avec des victimes, dont l'analyse est en cours (Robert, Zauberman, Levy, en cours). 
gences des compagnies d'assurance en matière d'équipements de protection, notamment pour certains locaux commerciaux).

Enfin, et surtout, cette solution n'efface pas le sentiment d'agression résultant de l'infraction, notamment lorsqu'elle frappe le domicile ${ }^{20}$, sentiment peut-être d'autant plus intense que le coupable reste non identifié. Classiquement, le recours au pénal s'est construit comme un élément de négociation ou de pression, dans le cadre d'une relation sociale entre des protagonistes qui se connaissent, comme constituant essentiellement un moyen d'aboutir à un compromis $^{21}$. Ce modèle traditionnel est brisé par l'incapacité de l'État à répondre à la plainte par l'identification de l'auteur. On se trouve alors dans une situation de mort du lien social qui autorise tous les fantasmes pour tenter de la conjurer et qui, aussi, peut paraître particulièrement menaçante pour les membres de couches sociales anxieuses de leur avenir.

Ne disposer que de recherches parcellaires sur la question permet bien de relever l'existence de tels traits, mais interdit d'en réaliser un examen différentiel, c'est-à-dire de préciser quels sont les groupes sociaux pertinents qui en sont porteurs. Cette situation présente le grave danger d'inciter parfois à des généralisations hâtives, qui nient la démarche sociologique.

\section{GÉRER L'INSÉCURITÉ?}

Schématiquement, on trouve quatre interprétations du sentiment d'insécurité22. La première, purement «substantialiste», l'explique par une augmentation de la délinquance, et c'est grosso-modo le thème sur lequel brodent traditionnellement les commentaires des autorités de police. Cette thèse a évidemment peu de crédit scientifique, dans la mesure où le problème gît tout entier non dans la délinquance, mais dans la représentation que l'on s'en fait. De surcroît, même les sondages commerciaux n'apportent pas beaucoup de crédit à cette thèse, puisqu'ils font traditionnellement apparaître la discordance entre peur du crime et expérience ${ }^{23}$.

20. En fait cette critique apparaît souvent sous deux formes: d'une part, le remboursement d'un objet ne compense pas, chez certaines personnes, le sentiment de perte lié à la valeur affective du bien en question; d'autre part, l'indemnisation est impuissante à éliminer le sentiment de viol de l'intimité que crée, chez certains, l'intrusion des voleurs (voir Dulong, 1981, p. 215, en particulier).

21. Castan, N. (1980, chap. I), Castan, N et Y. (1977, 1981), Soman (1980).

22. Pour une revue d'ensemble, voir Zauberman (1982c).

23. Peyrefitte (1977, I, p. 47 à 49). 
La deuxième interprétation est, au contraire purement «constructionniste»: elle affirme que l'insécurité est le fruit absolument gratuit d'une campagne d'opinion. Un temps soutenue par les milieux et la presse d'extrême-gauche, cette position, qui prête beaucoup aux gouvernants et à la presse, est maintenant à peu près totalement abandonnée, notamment au profit d'une théorie de la «dérivation artificięlle».

Dans cette troisième optique, le sentiment d'insécurité préexiste à la campagne d'opinion, mais il a d'autres causes que la délinquance, et c'est la campagne en question qui le rabat sur celle$\mathrm{ci}^{24}$. Néanmoins, ce n'est que pendant un laps de temps relativement bref que le discours public a embouché sans réserve la trompette de l'insécurité. Pratiquement jusqu'en 1978, il était traversé de contradictions manifestées par la traditionnelle divergence de vue entre les ministères de la Justice et de l'Intérieur, et depuis 1981 des divisions ont ressurgi dans le discours officiel à propos de la sécurité.

C'est pourquoi l'hypothèse la plus récente est celle de la cristallisation, bien fondée sur la délinquance, d'un sentiment d'insécurité dont le spectre d'origine est beaucoup plus large: les caractéristiques de la gestion de la délinquance fournissent un exemple, particulièrement congruent à ces inquiétudes, d'un phénomène redouté par ailleurs, par exemple le retrait de l'État vis-à-vis de certaines couches sociales habituées à sa sollicitude particulière ${ }^{25}$.

Dans cette hypothèse, certaines couches sociales ou fractions de classes, qui craignent d'être abandonnées par l'État, trouvent dans la gestion de la délinquance ${ }^{26}$ une excellente illustration de cette appréhension, sur laquelle elles peuvent cristalliser leur peur. Dans cette explication, la délinquance n'est pas simplement un exutoire, mais une concrétisation de l'insécurité.

24. Parmi les travaux français, Coing et Meunier (1980) sont particulièrement représentatifs de cette position. Certains auteurs (par ex. Verneuil (1981), pour la France, s'inspirant à travers D'Anjou et al. (1978), de Ranulf (1964), en présentent une version un peu différente: c'est spontanément que les groupes en proie à l'insécurité économique, tendent à lui trouver un objet de cristallisation aisée.

25. Dulong (1981 et 1983).

26. L'assignation à l'État de la mission de garantir la sûreté des personnes et des biens fait partie d'une idéologie bourgeoise que l'on peut supposer largement reçue sur ce point, même s'il serait imprudent de postuler sa généralisation à toutes les classes sociales. En France, cette croyance est peut-être d'autant plus ancrée qu'on peut lui trouver de très anciennes origines: dès la fin du Moyen-Âge, le roi apparaît investi d'une "garde générale» du royaume, qui a servi de base idéologique à l'organisation de la fonction pénale de la monarchie absolutiste. 
Au-delà de cette constatation très générale, à laquelle se bornent communément les défenseurs de cette thèse, on peut noter qu'elle est singulièrement renforcée par l'observation faite ci-dessus, de la non-élucidation tendancielle des plaintes des particuliers ${ }^{27}$.

Cette inefficacité n'est d'ailleurs pas dissimulée par la police et les plaignants la perçoivent très clairement. L'importance du phénomène est curieusement restée inaperçue, probablement parce qu'il ne paraît pas avoir entraîné jusqu'à présent une crise de confiance généralisée envers la police. Mais il faut bien comprendre que cette situation paradoxale tient à l'existence de deux contre-feux efficaces ${ }^{28}$.

D'abord, un plaidoyer sur le manque de moyens d'autant mieux reçu que les plaignants peuvent souvent constater par euxmêmes la vétusté des locaux et la faiblesse des effectifs affectés aux services de police auxquels ils s'adressent ${ }^{29}$.

Ensuite l'accusation de laxisme judiciaire, qui consiste à transférer dans le champ de l'intensité de la répression le débat qui pourrait naître dans celui de sa certitude, c'est-à-dire de l'efficacité de la police $^{30}$.

Toutefois, l'immunité policière, qui naît de l'efficacité de ces contre-feux, ne résout pas la revendication de sécurité, et l'on peut faire l'hypothèse que la délégitimation est transférée de la police à l'État tout entier. La politique suivie par le gouvernement de 1978 à 1981 y a probablement contribué: en affirmant à la fois la croissance d'une insécurité qui serait due au laxisme de sa justice et sa capacité à restaurer la sécurité, il a pris le risque que seule la première partie du message soit entendue, confirmant par là, involontairement, les soupçons que les groupes en proie à l'insécurité nourrissaient à l'endroit de l'État ${ }^{31}$.

27. Voy. déjà Robert et Faugeron, 1980, 142 et 199.

28. Ces éléments de l'interaction entre policiers et plaignants apparaissent notamment dans l'enquête en cours auprès des victimes (Robert, Zauberman, Levy, en cours).

29. Cette situation contraste cependant avec la très forte densité policière française (Commission des maires, 1982, p. 163). Elle traduit donc plutôt les choix dans l'allocation des ressources qu'un manque absolu de moyens.

30. Cet argument est également difficiement soutenable: par définition il ne peut y avoir laxisme ou excès de sévérité pour les contentieux que la police ne traite pas effectivement. Du reste, il est contredit par l'évolution depuis 1976 des indicateurs sur le recours à la détention provisoire et aux peines d'emprisonnement.

31. Cette délégitimation de l'État se traduit notamment par une appétence jamais satisfaite de répression (particulièrement étudiée par Verneuil, 1981, p. 226 et 233). 
Face à cette situation difficile, les gouvernements ont adopté une conduite assez erratique.

En 1977 encore, le Comité Peyrefitte ${ }^{32}$ s'en tenait au vieux discours prévention-réinsertion, sans du tout voir le problème posé à l'État par l'ineffectivité de sa police.

La politique suivie de 1978 à 1981 consistait surtout en une manipulation juridique destinée à laisser penser que la répression accrue de certaines infractions était de nature à désarmer l'insécurité. Là encore, la liaison entre la cristallisation de cette dernière sur la délinquance et les pratiques policières n'était pas analysée.

Concurremment à ces différentes politiques, on observe une croyance constante de tous les gouvernements dans l'efficacité d'une réponse purement quantitative qui se borne à accroître les effectifs policiers, sans s'interroger sur la possibilité de rendements décroissants, ni sur les priorités dans le recrutement, ni sur l'éventualité d'une refonte totale de la doctrine d'emploi de la police ${ }^{33}$. D'où une politique policière réduite à la multiplication, au coup par coup, d'unités spécialisées ${ }^{34}$ dont aucune, du reste, ne vise le contentieux ordinaire de plainte.

Le gouvernement actuel semble persuadé de l'inanité des modifications juridiques créant des régimes spéciaux à telle ou telle criminalité $^{35}$; en revanche, il n'a que très peu réalisé les problèmes posés par la doctrine d'emploi de la police ou, du moins, les amorces de prise de conscience ${ }^{36} n^{\prime}$ 'ont pas été relayées par une volonté politique capable de plier les résistances de la hiérarchie policière.

Par ailleurs, l'actuel gouvernement a tenté d'amorcer une redistribution des responsabilités entre pouvoir central et collectivités locales ${ }^{37}$. Si cette initiative se développait, elle constituerait peutêtre le point le plus original de la police actuelle: elle pourrait en effet

32. Peyrefitte (1977).

33. Sur ce point, le gouvernement actuel a du mal à se séparer clairement de ses prédécesseurs.

34. Spécialisées dans la détection de certaines délinquances: équjpes de répression du vol à la tire, unités de surveillance du métro parisien, etc.

35. Plus d'ailleurs par hostilité de juriste envers des lois d'exceptions que par claire conscience de leur inefficacité.

36. Belorgey (1982), Commission des maires (1982).

37. En constitutant une «Commission des maires sur la sécurité», puis en entérinant ses recommandations. 
fragmenter jusqu'à un certain point la revendication de sécurité et, partant, freiner le processus de perte de crédit de l'État central. Et d'ailleurs ce résultat pourrait être facilité si les maires, impliqués dans la gestion de la délinquance, ne trouvaient plus d'intérêt à relayer le sentiment d'insécurité, mais se voyaient plutôt conduits à tenter de le neutraliser. Néanmoins, il ne faut probablement pas voir là une stratégie consciente à long terme; plus vraisemblablement, cette initiative est née du souci de ne pas modifier la loi du 2 février 1981, dite «Sécurité et Liberté», sans avoir fait un geste à l'égard des maires des grandes villes. Du reste, une claire prise de conscience de ces enjeux est d'autant plus improbable que cette orientation se heurte au jacobinisme de toutes les administrations de l'État centra $^{38}$. Les hésitations dans la mise en cuvre des recommandations de la commission des maires semblent d'ailleurs bien confirmer qu'il n'y a pas là de stratégie claire.

Contrairement à ce que beaucoup ont affirmé, l'attitude du gouvernement sous le précédent septennat a été plus hésitante que machiavélique en matière pénale et le gouvernement actuel a eu du mal à adopter une démarche plus cohérente comme le montre la contrariété des attitudes du ministère de l'Intérieur et de celui de la Justice.

La faillite de l'État en ce domaine est, semble-t-il, aux yeux de certains groupes sociaux, habitués à chercher dans sa protection la marque de leur alliance avec les fractions dominantes, la preuve de la rupture de celle-ci ... rupture que d'autres éléments d'une autre ampleur les amenaient déjà à soupçonner plus ou moins confusément.

Dans les travaux existants, deux pistes, éventuellement cumulatives, sont évoquées pour identifier les groupes en cause.

C'est d'abord, bien entendu, la petite bourgeoisie non salariée traditionnelle ${ }^{39}$ - celle de la boutique - à qui son alliance traditionnelle avec la bourgeoisie a longtemps valu une particulière protection étatique. Depuis plus de vingt ans, cette fraction de classe résiste avec vigueur à la concentration de circuits de distribution dont l'archaïsme a toujours protégé sa rente de situation.

38. Sauf peut-être la Justice qui est la moins centralisée et qui, compte tenu de sa forte délégitimation, a peu à y perdre.

39. Voir notamment les travaux précités de Dulong $(1981,1983)$. 
C'est aussi, peut-être une fraction de la petite bourgeoisie salariée, notamment d'État, qui subit un processus relatif de déqualification et de perte de prestige social: le cas de figure des instituteurs est particulièrement typique.

C'est en ce sens que l'on recourt à l'hypothèse d'un mouvement «poujadiste» ${ }^{40}$, au sens précis que revêt cette expression dans la science politique française, c'est-à-dire comme une protestation adressée à l'État par un groupe social, contre son abandon par ce même État ${ }^{41}$.

Par ailleurs, plusieurs indices ${ }^{42}$ conduisent à se demander s'il n' existe par également, au sein, cette fois, de certaines fractions de la classe dominante, un mécontentement envers ce qui apparaît comme un laxisme de l'État. Dans ce cas on ne pourrait plus parler de peur ou d'un sentiment d'insécurité personnel, mais ce mécontentement rejoindrait néanmoins la revendication angoissée de certaines fractions petites bourgeoises.

En l'absence de travaux suffisamment nombreux et documentés, il n'est malheureusement possible ni de tester cette hypothèse séduisante, ni de préciser, au-delà du simple vraisembable, quels groupes sociaux elle affecterait ${ }^{43}$.

Ce constat récurrent d'une absence de données permettant une analyse différentielle des réactions à la pratique policière, montre bien qu'une véritable sociologie de la police en France reste à bâtir.

Si l'on veut y parvenir, il est d'abord nécessaire de procéder à un sérieux travail de construction de l'objet, qui soit en premier lieu une déconstruction des mythes habituels, notamment de plus prégnant, celui qui couple l'imputation de machiavélisme à la croyance

40. Du nom du chef d'un mouvement de petits commerçants au milieu des années cinquante (Pierre Poujade).

41. Dulong (1981, p. 218), Robert (1982).

42. Des travaux sur les priorités relatives de répression pénale (voir Robert et Faugeron, 1980 , p. 181) montrent que les fractions non salariées de la bourgeoisie se retrouvent avec les petits commerçants et les artisans pour souhaiter en priorité une répression des atteintes à la propriété individuelle. Par ailleurs, la préoccupation envers le problème criminel apparaît, d'après une campagne d'entretiens en cours avec des victimes, comme un phénomène particulièrement typique de la bourgeoisie industrielle et commerciale (Robert, Zauberman, Levy, en cours).

43. Ce dernier point est rendu peut-être plus difficile désormais par l'effet de contamination qu'ont pu produire la persistance et la vivacité du débat public autour de l'insécurité. 
que le développement quantitatif de l'appareil policier s'accompagne inéluctablement d'un surcroît d'efficacitét ${ }^{44}$.

Il sera ensuite indispensable de rassembler et d'organiser des données (sur l'évolution des effectifs, les dotations, l'origine sociale des policiers, leur productivité...) qui sont éparses, mais que l'on peut souvent reconstituer sur longue période.

Enfin, l'exigence d'analyse différentielle interdit d'importer imprudemment des modèles d'analyses construits dans des contextes sociaux et institutionnels différents et contraint à éviter les généralisations abusives et à tenter de préciser à chaque fois qui fait ou pense quoi.

\section{BIBLIOGRAPHIE}

BARBERGER-DAMAMME, C. (1981), De la criminalité apparente, Lyon, Université Jean Moulin.

BELORGEY, J.M. (1982), Pré-rapport du parlementaire en mission sur les problèmes de police, Paris, ministère de l'Intérieur et de la Décentralisation (inédit).

BONNEMAIN, C. (1978), Le contrôle social de la déviance, recherche au niveau d'un service de police, Paris, Poitiers, Centre d'Études sociologiques, Université de Poitiers.

BOURDIEU, P. (1980), «Le mort saisit le vif. Les relations entre l'histoire réifiée et 'histoire incorporée», Actes de la recherche en sciences sociales, p. 32-33 et p. 4-14.

CASTAN, N. (1980), Justice et répression en Languedoc à l'époque des Lumières, Paris, Flammarion.

CASTAN, N. et Y. CASTAN (1977), Recours, besoins et sens de la justice devant l'institution judiciaire française au xvill ${ }^{\mathfrak{e}}$ siècle, Amsterdan, Communication au $1^{\text {er }}$ colloque de l'I.A.H.C.C.J. (inédit).

CASTAN, N. et Y. CASTAN (1981), Vivre ensemble, ordre et desordre en Languedoc (XVIt ${ }^{2}$ XVII $f^{\prime}$ siècles), Paris, Gallimard, Julliard.

COING, H. et C. MEUNIER (1980), Insécurité urbaine? Une arme pour le pouvoir, Paris, Anthropos.

COMMISSION DES MAIRES SUR LA SÉCURITÉ (1982), Face à la délinquance: prévention, répression, solidarité, Paris, Documentation française.

44. On pense à la mise en garde de Bourdieu $(1980$, p. 4) qui raille à la fois la propension à chercher un Deus in machine et celle à lire les conduites dans la description des appareils. 
D'ANJOU, L.J.M., C. COZIJN, L.D.V. TOORN, C.N. VERKOEYEN (1978), «Demanding More Severe Punishment», British Journal of Criminology, vol. $18, n^{\circ} 4$, p. $326-347$.

DIRECTION DE LA RÉGLEMENTATION, État annuel comparé des infractions à la police de la circulation, Paris, ministère de l'Intérieur (inédit).

DULONG, R. (1981), «Sens et non-sens de l'autodéfense», Déviance et Société, vol. $5, \mathrm{n}^{\circ} 3$, p. $211-222$.

FAUGERON, C., M. FICHELET, Ph. ROBERT (1977), Le renvoi du déviant, $\mathrm{Pa-}$ ris, C.O.R.D.E.S.

FRANCE. MINISTÈRE DE L'INTÉRIEUR, La criminalité en France d'après les statistiques de police judiciaire, Paris, Documentation française (annuel, 1973 à 1982).

GODEFROY, T. et B. LAFFARGUE (1982), Le coût du crime en France (19781979), Paris, S.E.P.C.

LE CLERE, M. (1980), Bibliographie critique de la police, Paris, YZER.

LEVY, R. (1982), Les «flags», une justice ou une police?, Paris, S.E.P.C.

LEVY, R. (1983), Les flagrants délits (titre provisoire), Bordeaux, Université Bordeaux 1 (sous presse).

PEYREFITTE, A. (édit.) (1977), Réponses à la violence, Paris, Presse Pocket.

RANULF, S. (1964), Moral Indignation and Middle-Class Psychology, New York, Schocken books.

ROBERT, $\mathrm{Ph}$. (1982), «Insécurité et délinquance», NON! Repères pour le socialis$m e$, vol. 14, p. 76-79.

ROBERT, Ph. et R. LEVY, Histoire et question pénale (sous presse).

ROBERT, Ph. et C. FAUGERON (1980), Les forces cachées de la justice, Paris, Centurion.

ROBERT, Ph., R. ZAUBERMAN, R. LEVY, Victimes d'infractions et justice pénale. Attitudes et comportements en cas de victimisation, Paris, S.E.P.C. (recherche en cours).

SERVERIN, E. et S. BRUXELLES (1981), Action et interactions dans l'institution judiciaire, Lyon, Université Jean Moulin (2 vol).

SHEARING, C.D. et P.C. STENNING (1983), «Private Security: Implications for Social Control», Social Problems, vol. 30, nº S, p. 493-506.

SOMAN, A. (1980), «Deviance and Criminal Justice in Western Europe, 1300-1800: An Essay in Structure», Criminal Justice History, vol. 1, p. 3-28.

VERNEUIL, D. (1981), «Fonctions et processus du système pénal: ce qu'on en dit dans le public", Déviance et Société, vol. 5, n 3, p. 223-245.

ZAUBERMAN, R. (1982a), Trajectoires de la déviance. Le renvoi des mineurs à la justice, Paris, S.E.P.C.

ZAUBERMAN, R. (1982b), «Renvoyants et renvoyés», Déviance et Société, vol. 6, $n^{\circ} 1$, p. 23-52.

ZAUBERMAN, R. (1982c), «La peur du crime et la recherche», Année sociologique, vol. 32 , p. $415-438$. 